\title{
DIFFUSION MAGNETIC RESONANCE IMAGING IN TRANSIENT GLOBAL AMNESIA
}

\author{
Clecio Godeiro-Junior', Maramélia Araújo de Miranda-Alves', Ayrton Roberto Massaro²
}

Transient global amnesia (TGA) is a well known clinical entity characterized by anterograde memory disturbance of sudden onset that lasts 1 to 24 hours. Orientation in space and time is impaired while consciousness remains undisturbed ${ }^{1,2}$. TGA may refer to a single expression of several physiopathological phenomena. Conceptually, cerebral ischemia, epileptic discharge, and migraine constitute the main pathogenic hypothesis ${ }^{3}$.

Diffusion-weighted imaging (DWI) has become a powerful tool in the evaluation of patients with suspected stroke owing to its high sensitivity and specificity, even for small areas of acute ischemia. Consequently, this method has also been applied to TGA to gain further insights into the ischemic hypothesis of this condition ${ }^{4}$.

We report a patient with a typical TGA presentation and MRI findings suggestive of an ischemic insult. We further discuss the ischemic hypothesis of TGA.

\section{CASE}

A 75-year-old female patient with past history of arterial hypertension and dyslipidemia was admitted to the emergency room presenting sudden behavioral changes starting eight hours before admission, approximately $2 \mathrm{pm}$. The patient's daughter accompanied her throughout the whole event and described the symptoms. While in the emergency room, she became agitated and disoriented, spoke fluently but did not recognize her family and repeatedly asked where she was and the time. Her neurological examination was unremarkable except for the behavioral disturbance and time-space disorientation. She had had two similar episodes with sudden onset and complete recovery after less than 6 hours, three years and one year before. In none of these episodes an exacting trigger was identified.

Laboratory testing at admission (blood counts, biochemistry, electrolytes, and coagulogram), electrocardiogram (ECG), and brain computerized tomography (CT) scan were normal. She recovered spontaneously 18 hours after the onset of clinical symptoms, but the patient was not able to recall any prior events. Carotid and vertebral arteries duplex scan, transcranial doppler ultrasonography, and transthoracic echocardiogram were unremarkable. MRI, performed 33 hours after clinical onset, showed high-intensity signal abnormality in DWI sequence in the left mesial temporal region (Figure). Based on her risk factors for stroke and neuroimaging findings, she was discharged on antiplatelet therapy. An electroencephalogram (EEG) performed during outpatient follow-up was normal.

We received the informed consent from the patient for this publication.

\section{DISCUSSION}

The clinical features of our patient meet the criteria of TGA and the high-intensity abnormality in left temporal region in DWI sequence points to an ischemic etiology. Our patient also presented risk factors for cerebrovascular disease: age, arterial hypertension and dyslipidemia. However, its reversibility and the nonexistence of other neurological signs are opposing arguments to this hypothesis. In fact, the ischemic hypothesis is very controversial.

According to the ischemic hypothesis, amnesia might result from a reversible dysfunction of frontotemporal structures caused by hemodynamic or thromboembolic ischemic mechanisms affecting the posterior cerebral and anterior choroideal arteries, and recurrent artery of Heubner. In a series of 26 Brazilian patients with TGA, risk factors for cerebrovascular disease were found in 13 cases, mainly hypertension and diabetes. This study was per-

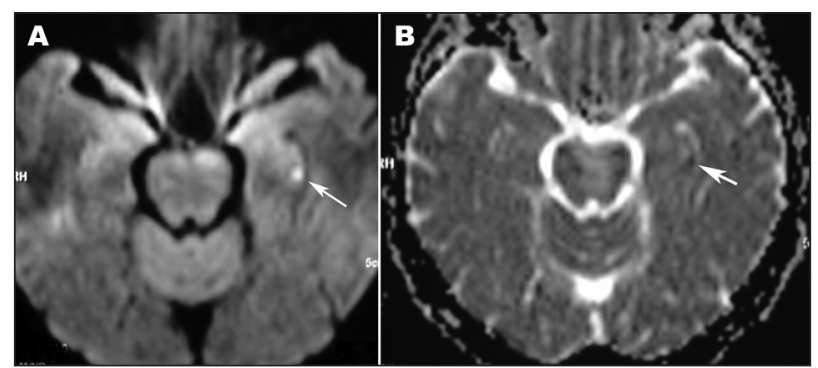

Figure. Brain MRI, carried out after 33 hours of the beginning of the symptoms. The axial sequence with diffusion $(A)$ and the map ADC (B) demonstrate a small hyperintense focus, with restriction to the diffusion (arrows), in the left anterior hipocampal region.

\section{RESSONÂNCIA MAGNÉTICA COM DIFUSÃO NA AMNÉSIA GLOBAL TRANSITÓRIA}

'Department of Neurology and Neurosurgery, Federal University of São Paulo, São Paulo SP, Brazil; ${ }^{2}$ Fleury Diagnostic Center, São Paulo SP, Brazil.

Received 25 July 2008, received in final form 6 October 2008. Accepted 19 November 2008.

Dr. Clecio Godeiro-Junior - Rua Dr. Diogo de Faria 650 / 33 - 04037-002 São Paulo SP - Brasil.E-mail: cleciojunior@yahoo.com.br 
formed in a period before the current use of DWI. In this study, brain angiography showed abnormalities in the vertebro-basilar system arteries in 3 patients, and CT showed ischemia in the vertebro-basilar system in only $2^{5}$. In more recent DWI studies, it has been demonstrated hyperintense lesions in the hippocampal region and in structures of the limbic system in TGA patients, comparable to ischemic stroke patients ${ }^{6,7}$. These hyperintense hippocampal lesions have small size (1 to $2 \mathrm{~mm}$ diameter), low apparent diffusion coefficient (ADC) and generally resolve in two weeks. However, these imaging abnormalities in TGA patients, although found in ischemia of arterial origin, are not specific and may also occur due to prolonged ictal activity, multiple sclerosis, hypoglycemia, venous thrombosis and phenilcetonuria ${ }^{7,8}$.

TGA patients usually have lower prevalence of cerebrovascular risk factors compared to stroke patients, as well as better prognosis, lower recurrence and later onset of lesions in imaging studies ${ }^{8}$. In a recent study, the vascular risk profile of TGA patients and concomitant changes on brain MRI were comparable with those of healthy controls and did not show significant differences between those with (DWI+) and without (DWI-) DWI lesions. A comprehensive diagnostic workup also provided no evidence for a higher rate of cerebrovascular disorder-related abnormalities in either the total group of TGA patients or TGA DWI+ patients. These findings do not support a cerebrovascular etiology of TGA, even in those individuals showing acute DWI lesions ${ }^{4}$. In another report, the topography and time course of hippocampal DWI lesions were studied in 41 TGA patients. Of those, 29 showed DWI lesions in the hippocampus within a time window of 48 $\mathrm{h}$ after onset. Almost all lesions (94\%) were selectively found in the CA-1 sector of the hippocampal cornu Ammonis. A follow-up study 4-6 months after the episode did not show evidence for residual structural sequel of these lesions ${ }^{9}$. The lack of sequel lesions is a strong argument against ischemic etiology. But, in opposition to this previous report, it has been recently demonstrated the presence of hippocampal cavitary lesions, specifically in CA1 region, in TGA patients, which is suggestive of late neuronal loss in this area ${ }^{10}$. Due to these contradictory findings, the true etiology of DWI lesions in the context of TGA remains speculative.

To complicate this discussion, it has been formulated the venous congestion hypothesis, based on the observation of Vasalva-like activities such as sexual intercourse and coughing preceding TGA episodes ${ }^{11,12}$. A study using duplex ultrasonography and magnetic resonance angiography (MRA) had verified a higher prevalence of valvular incompetence of the internal jugular vein in TGA patients compared to normal controls. These results lend support to the hypothesis that TGA may be attributable to venous congestion, and consequent venous ischemia of hippocampal structures ${ }^{12}$. Thus, it seems that there is not a unique vascular mechanism to explain TGA. As a symptom complex rather than a specific disease entity, TGA could result from any process that transiently disrupts memory while sparing other cognitive functions, so we are probably wasting time looking for a single cause ${ }^{13}$.

We believe that a DWI lesion in TGA patient has to be considered non-specific finding with different possible underlying mechanisms, probably all leading to focal energy failure. Thus, vascular and non-vascular causes require further investigation. Meanwhile, avoidance of and correction of cerebrovascular risk factors are certainly appropriate in any individuals.

ACKNOWLEDGEMENT - We would like to thank Doctor Suzana Malheiros for reviewing this text.

\section{REFERENCES}

1. Pantoni L, Lamassa M, Inzitari D. Transient global amnesia: a review emphasizing pathogenic aspects. Acta Neurol Scand 2000;102:275-283.

2. Sander K, Sander D. New insights into transient global amnesia: recent imaging and clinical findings. Lancet Neurology 2005;4:437-444.

3. Quinette P, Guillery-Girard B, Dayan J, et al. What does transient global amnesia really mean? Review of the literature and thorough study of 142 cases. Brain 2006;129:1640-1658.

4. Enzinger C, Thimary F, Kapeller P, et al. Transient global amnesia: diffusion-weighted imaging lesions and cerebrovascular disease. Stroke 2008;39:2219-2225.

5. Zukerman E, Andrade LA, Bertolucci PH. Transient global amnesia study of 26 cases. Arq Neuropsiquiatr 1985;49:39-47.

6. Sedlaezek O, Hirsch JG, Grips E, et al. Detection of delayed focal MR changes in the lateral hippocampus in transient global amnesia. Neurology 2004;62:2165-2170.

7. Tong DC, Grossman M. What causes transient global amnesia? New insights from DWI. Neurology 2004;62:2164-2165.

8. Winbeck K, Etgen T, von Einsiedel HG, Rottinger M, Sander D. DW in transient global amnesia and TIA: proposal for an ischemic origin of TGA. J Neurol Neurosurg Psychiatry 2005;76:438-441.

9. Bartsch T, Alfke K, Stingele R, et al. Selective affection of hippocampal CA-1 neurons in patients with transient global amnesia without longterm sequel. Brain 2006; 129:2874-2884.

10. Weon YC, Kim JH, Lee JS, Kim SY. Optimal diffusion-weighted imaging protocol for lesion detection in transient global amnesia. AJNR Am J Neuroradiol 2008;29:1324-1328.

11. Schreiber SJ, Doepp F, Klingebiel R, Valdueza JM. Internal jugular vein valve incompetence and intracranial venous anatomy in transient global amnesia. J Neurol Neurosurg Psychiatry 2005;76:509-513.

12. Sander D, Winbeck K, Etgen T, Knapp R, Klingelhöfer J, Conrad B.. Disturbance of venous flow patterns in patients with transient global amnesia. Lancet 2000;357:957.

13. Roach ES. Transient global amnesia: look at mechanisms not causes Arch Neurol 2006;63:1338-1339. 